$\underline{\mathbf{P}-178}$

\title{
Synthesis of Peptides Based on Betulinic Acid Scaffold
}

\author{
Mohd Tajudin Mohd Ali*, Habsah Zahari and Siti Aisyah Aliasak \\ Fakulty of Applied Sciences, Universiti Teknologi MARA, 40450 Shah Alam, Malaysia; \\ E-mail: tajudinali@salam.uitm.edu.my
}

Triterpene acid compounds are potent anti-tumor agents showing a remarkable cytotoxicity on various tumor cells. Betulinic acid (BA), as the main constituents of Melaluca cajiput sp. bark is one of the promising compound that also has anti-retrovial, anti-malaria, anti HIV and anti-inflammatory activities. Based on theirs therapeutic properties of peptide based on betulinic acid, we became interested to synthesized the betulinic acid peptides. We herein report the synthesis of several natural amino acid-BA peptides using standard coupling protocols (HOBt, HBTU, DiPEA). In addition, enantiopure cyclohexene silyl etherBA peptide, was successfully synthesized. The result of the biological evaluation are expected to improve the general understanding of BA structure.

Keywords: Azido cyclohexene silyl ether, peptides, betulinic acid. 